\title{
Hopf Bifurcation Analysis in a Modified Price Differential Equation Model with Two Delays
}

\author{
Yanhui Zhai, Ying Xiong, and Xiaona Ma \\ School of Science, Tianjin Polytechnic University, Tianjin 300387, China \\ Correspondence should be addressed to Ying Xiong; 542790840@qq.com
}

Received 14 November 2013; Accepted 13 January 2014; Published 3 March 2014

Academic Editor: Weiming Wang

Copyright ( 2014 Yanhui Zhai et al. This is an open access article distributed under the Creative Commons Attribution License, which permits unrestricted use, distribution, and reproduction in any medium, provided the original work is properly cited.

\begin{abstract}
The paper investigates the behavior of price differential equation model based on economic theory with two delays. The primary aim of this thesis is to provide a research method to explore the undeveloped areas of the price model with two delays. Firstly, we modify the traditional price model by considering demand function as a downward opening quadratic function, and supply and demand functions both depending on the price of the past and the present. Then the price model with two delays is established. Secondly, by considering the price model with one delay, we get the stable interval. Regarding another delay as a parameter, we studied the linear stability and local Hopf bifurcation. In addition, we pay attention to the direction and stability of the bifurcating periodic solutions which are derived by using the normal form theory and center manifold method. Afterwards, the study turns to simulate the results through numerical analysis, which shows that the provided method is valid.
\end{abstract}

\section{Introduction}

Recent developments in mathematical economics and in problems of business administration have led to extensive use of differential equation model. Bifurcations and chaos always show in the contemporary literature of economics as basic concepts. The paper of Shuhe [1] was the pioneering work in studying price differential equation model which provides a dynamic system to investigate sectoral dynamics of an economy phenomenon. Since demand depends on the past price, further study on price model with delay can be found in [2]. Reference [3] focused on the phenomenon of bifurcation which forms an integral part of qualitative approach to study dynamical systems. Similarly, [4-6], which investigated the local Hopf bifurcation and the existence of periodic solutions of price model, had important consequences for theoretical and empirical model building in economics. Reference [7] provided a brief survey of the literature of bifurcation detections in economic models. The existence of different bifurcate parameters leads to chaos and the causes of complicated phenomenon were argued in [8]. In short, few people studied price model with delay; what is more, no results involving price model with two delays have occurred.
In order to illustrate the economic phenomena with price varying accurately, a reasonable mathematical model of price is needed. Thus, we introduce a traditional price differential equation model in [1]:

$$
\frac{d^{2} P(t)}{d t^{2}}=\mu \delta(P(t)) \frac{d P(t)}{d t}-\mu b_{0} P(t)+\mu d_{0}-\mu g_{0} .
$$

The meaning of parameters refers to [1]. We modify the traditional price differential equation model by considering the following factors.

Firstly, we denote the correlation coefficient between demand and price rising rate by $\delta(P(t))$. We consider demand function as a downward opening quadratic function and supply function as a linear function:

$$
\delta(P(t))=b(P(t)+\beta)^{2}+C_{0}, \quad b<0, \beta<0, C_{0}<0 .
$$

Secondly, according to the cobweb theory, since manufacturers need a production cycle time from obtaining market information to adjust the production line, the role of price adjustment lags on the supply function of time. The purchase of the consumers also depends on the price change and 
decision whether to buy or not. Then, supply and demand functions both depend on the price of the past and the present. We introduce two delays $\tau_{1}, \tau_{2}$ to denote supply and demand functions, which depend on the price of the past, respectively. A differential system with two delays for price differential equation model is transformed into the following form:

$$
\begin{aligned}
\frac{d^{2} P(t)}{d t^{2}}= & \mu\left[b(P+\beta)^{2}+C_{0}\right] \frac{d P\left(t-\tau_{2}\right)}{d t} \\
& -\mu b_{0} P(t)-\mu a P\left(t-\tau_{1}\right)+\mu\left(d_{0}-g_{0}\right) .
\end{aligned}
$$

Let $P(t)=x(t), d P(t) / d t=y(t)$, and notice that supply and demand functions both depend on the price of the past and the present; then the model is described by the following autonomous system:

$$
\begin{gathered}
\dot{x}(t)=d y(t)+k y\left(t-\tau_{2}\right), \\
\dot{y}(t)=\mu\left[b(x(t)+\beta)^{2}+C_{0}\right] y\left(t-\tau_{2}\right)-\mu b_{0} x(t) \\
-\mu a x\left(t-\tau_{1}\right)+\mu\left(d_{0}-g_{0}\right),
\end{gathered}
$$

where $d, k$ refers to [2]. $x(t)$ is the price at time $t$, and $y(t)$ is the amount of supply at time $t ; \mu>0, b_{0}>0, a>0, b<0$, $\beta<0, C_{0}<0$.

Different from the previous work in $[1,2,4]$, the purpose of this paper is to investigate the stability of the Hopf bifurcation and the direction of bifurcation periodic solution of a price differential equation model with two delays. The structure of the paper is as follows. In Section 2, linear stability and local Hopf bifurcations are studied by using qualitative methods. In Section 3, we regard $\tau_{2}$ as bifurcation parameter and consider (4) with $\tau_{1}$ in its stable interval; the direction of Hopf bifurcation and the stability of the bifurcation periodic solutions are derived by using the normal form theory and center manifold method. Afterwards, the presented numerical simulations in Section 4 have demonstrated the theoretical analysis.

\section{Local Stability and Hopf Bifurcation}

Obviously, system (4) always has an equilibrium $E^{*}=$ $\left(x^{*}, y^{*}\right)=\left(\left(d_{0}-g_{0}\right) /\left(b_{0}+a\right), 0\right)$. In the following, we will investigate the effect of the delay $\tau_{1}, \tau_{2}$ on the dynamics of system (4).

Let $u(t)=x(t)-x^{*}, v(t)=y(t)-y^{*}$; the linearization of system (4) at zero steady state is

$$
\begin{gathered}
\dot{u}(t)=d v(t)+k v\left(t-\tau_{2}\right), \\
\dot{v}(t)=\mu\left[b\left(P_{0}+\beta\right)^{2}+C_{0}\right] v\left(t-\tau_{2}\right) \\
-\mu b_{0} u(t)-\mu a u\left(t-\tau_{1}\right) .
\end{gathered}
$$

For convenience, as in literature of [5], we denote $A=$ $\left.-\mu\left[b\left(P_{0}+\beta\right)^{2}+C_{0}\right)\right]>0, B=\mu b_{0}>0, C=\mu a>0$. Then $(5)$ become

$$
\begin{gathered}
\dot{u}(t)=d v(t)+k v\left(t-\tau_{2}\right), \\
\dot{v}(t)=-A v\left(t-\tau_{2}\right)-B u(t)-C u\left(t-\tau_{1}\right) .
\end{gathered}
$$

The corresponding characteristic equation is

$$
\lambda^{2}+A e^{-\lambda \tau_{2}} \lambda+B k e^{-\lambda \tau_{2}}+C d e^{-\lambda \tau_{1}}+C k e^{-\lambda\left(\tau_{1}+\tau_{2}\right)}=0 .
$$

To study the stability of equilibrium $E^{*}$ of (4) and Hopf bifurcation, it is sufficient to analyze the distribution of the roots of (7). It is stable if all roots of (7) have negative real parts and unstable if one root has positive real part. In order to study the characteristic (7) with two delays, we first consider (7) with one delay. Without loss of generality, we choose $\tau_{1}$ as a parameter and employ Rouche's theorem in Cooke and Grossman [9]; we will find the stable interval for $\tau_{1}$. Then we consider (7) with $\tau_{1}$ in its stable intervals. Using Rouche's theorem again, regard $\tau_{2}$ as a parameter; we will find the stable interval (depends on $\tau_{1}$ ) for $\tau_{2}$. Then we obtain the stable interval for system (4).

Now we analyse the case when $\tau_{2}=0$; (7) becomes

$$
\lambda^{2}+A \lambda+B k+C d e^{-\lambda \tau_{1}}+C k e^{-\lambda \tau_{1}}=0 .
$$

Using a procedure similar to [10], we make some hypotheses as follows:

$$
\begin{aligned}
& \text { (H1) } A^{2} \leq 4[B k+C d+C k], \\
& \text { (H2) } A^{2}>4[B k+C d+C k], \\
& \text { (H3) } B^{2} k^{2}<C^{2}(d+k)^{2}, \\
& \text { (H4) } B^{2} k^{2}>C^{2}(d+k)^{2}, 2 B k>A^{2} \text {, and }\left(-2 B k+A^{2}\right)^{2}> \\
& 4\left[B^{2} k^{2}-C^{2}(d+k)^{2}\right],
\end{aligned}
$$

(H5) neither (H3) nor (H4).

Obviously, when $\tau_{1}=\tau_{2}=0$, (4) becomes a system of ODE. Under the hypothesis ( $\mathrm{H} 1)$, all roots of (7) have negative real parts if and only if $A>0$; under the hypothesis ( $\mathrm{H} 2)$, all roots of (8) have negative real parts if and only if $A>0$ and $B k+$ $C d+C k>0$. Above all, either $(\mathrm{H} 1)$ or $(\mathrm{H} 2)$ holds for $\tau_{1}=$ $\tau_{2}=0$, and all roots of (7) have negative real parts.

Applying the lemma in [9] again, we obtain the following results.

Lemma 1. For (8), one has the following:

(i) if (H3) holds and $\tau_{1}=\tau_{1, n}^{(1)}$, then (8) has a pair of purely imaginary roots $\pm i \omega_{+}$;

(ii) if (H4) holds and $\tau_{1}=\tau_{1, n}^{(1)}$ (res. $\tau_{1}=\tau_{1, n}^{(2)}$ ) then (8) has a pair of imaginary roots of $\pm i \omega_{+}$(res. $\left.\pm i \omega_{-}\right)$;

(iii) if (H5) holds and $\tau_{2}>0$, then (8) has no purely imaginary root, where

$$
\begin{aligned}
& \omega_{ \pm}^{2}= \frac{2 B k-A^{2}}{2} \\
& \pm\left[\frac{1}{4}\left(-2 B k+A^{2}\right)^{2}-B^{2} k^{2}+C^{2}(d+k)^{2}\right]^{1 / 2}, \\
& \\
& \tau_{1, n}^{(1)}=\frac{1}{\omega_{+}} \arccos \left\{\frac{\left(\omega_{+}^{2}-B k\right)}{C d+C k}\right\}+\frac{2 n \pi}{\omega_{+}},
\end{aligned}
$$

$$
\tau_{1, n}^{(2)}=\frac{1}{\omega_{-}} \arccos \left\{\frac{\left(\omega_{-}^{2}-B k\right)}{C d+C k}\right\}+\frac{2 n \pi}{\omega_{-}}, \quad(n=0,1, \ldots) .
$$


Proof. Let $\lambda=i \omega$ be the root of (8); we thus have

$$
-\omega^{2}+A \omega i+B k+C(d+k) e^{-\lambda \tau_{1}}=0 .
$$

Separating the real and imaginary parts

$$
\begin{gathered}
-\omega^{2}+B k+C(d+k) \cos \omega \tau_{1}=0, \\
A \omega-C(d+k) \sin \omega \tau_{1}=0 .
\end{gathered}
$$

Eliminating the harmonic terms gives

$$
\omega^{4}+\left(-2 B k+A^{2}\right) \omega^{2}+B^{2} k^{2}-C^{2}(d+k)^{2}=0 .
$$

Obviously

$$
\omega_{1}^{2} \cdot \omega_{2}^{2}=B^{2} k^{2}-C^{2}(d+k)^{2}, \quad \omega_{1}^{2}+\omega_{2}^{2}=2 B k-A^{2} .
$$

From (12) and by direct computation, we obtain $\omega_{ \pm}^{2}, \tau_{1, n}^{(1)}, \tau_{1, n}^{(2)}$. The result is straightforward.

Denote the minimum value of $\tau_{1, n}$ by $\tau_{1}^{0}$; that is, $\min \left(\tau_{1, n}\right)=\tau_{1}^{0}$, and

$$
\begin{aligned}
\lambda_{k, n} & =\alpha_{k, n}\left(\tau_{1}\right)+i \omega_{k, n}\left(\tau_{1}\right), \\
\alpha_{1, n}\left(\tau_{1, n}^{(1)}\right) & =0, \quad \omega_{1, n}\left(\tau_{1, n}^{(1)}\right)=\omega_{+}, \\
\alpha_{2, n}\left(\tau_{1, n}^{(2)}\right) & =0, \quad \omega_{2, n}\left(\tau_{1, n}^{(2)}\right)=\omega_{-} .
\end{aligned}
$$

To see if $\tau_{1, n}^{(1)}$ and $\tau_{1, n}^{(2)}$ are bifurcation values, we need to verify if the transversality conditions hold.

Lemma 2. The following transversality conditions

$$
\frac{d \operatorname{Re} \lambda_{1, n}\left(\tau_{1, n}^{(1)}\right)}{d \tau_{1}}>0, \quad \frac{d \operatorname{Re} \lambda_{1, n}\left(\tau_{1, n}^{(2)}\right)}{d \tau_{1}}>0,
$$

hold.

Proof. Differentiating (8) with respect to $\tau_{1}$ yields

$$
\begin{gathered}
2 \lambda \frac{d \lambda}{d \tau_{1}}+A \frac{d \lambda}{d \tau_{1}}+C(k+d) e^{-\lambda \tau_{1}}\left(-\tau_{1} \frac{d \lambda}{d \tau_{1}}-\lambda\right)=0 \\
\frac{d \lambda}{d \tau_{1}}=\frac{C(k+d) \lambda e^{-\lambda \tau_{1}}}{2 \lambda+A-C(k+d) \tau_{1} e^{-\lambda \tau_{1}}}
\end{gathered}
$$

then we have

$$
\begin{aligned}
\operatorname{Re} \frac{d \lambda}{d \tau_{1}} & =\operatorname{Re}\left\{\frac{2 \lambda+A}{C(k+d) \lambda e^{-\lambda \tau_{1}}}-\frac{\tau_{1}}{\lambda}\right\}=\frac{2 \lambda+A}{C(k+d) \lambda e^{-\lambda \tau_{1}}} \\
& =\frac{1}{C(d+k)} \cdot \frac{2 i \omega+A}{i \omega\left(\cos \omega \tau_{1}-i \sin \omega \tau_{1}\right)} \\
& =\frac{1}{C(d+k)} \cdot \frac{A \omega \sin \omega \tau_{1}+2 \omega^{2} \cos \omega \tau_{1}}{\omega^{2}},
\end{aligned}
$$

which satisfied $C>0$ and $d+k>0$; then for $\lambda_{1, n}\left(\tau_{1, n}^{(1)}\right), d \operatorname{Re} \lambda_{1, n}\left(\tau_{1, n}^{(1)}\right) / d \tau_{1}>0$, and for $\lambda_{1, n}\left(\tau_{1, n}^{(2)}\right)$, $d \operatorname{Re} \lambda_{1, n}\left(\tau_{1, n}^{(2)}\right) / d \tau_{1}>0$. We complete the proof.
(8).

Thus, we get the distribution of the characteristic roots of

Lemma 3. For (8), one has the following:

(i) if (H3) and either (1) (H1) A>0 or (2) (H2) A>0 and $B k+C d+C k>0$ hold, then when $\tau_{1} \in\left[0, \tau_{1,0}^{(1)}\right)$, all roots of (8) have negative real parts, and when $\tau_{1}>\tau_{1,0}^{(1)}$, (8) has at least one root with positive real part;

(ii) if (H4) and either (H1) or (H2) hold, then there are $k$ switches from stability to instability; that is, when $\tau_{1} \in$ $\left(\tau_{1, n}^{(2)}, \tau_{1, n+1}^{(1)}\right), n=-1,0,1, \ldots, k-1$, all roots of $(8)$ have negative real parts, where $\tau_{2,-1}^{(2)}=0, \tau_{1} \in\left[\tau_{1, n}^{(1)}, \tau_{1, n+1}^{(2)}\right)$ and $\tau_{1}>\tau_{1, k}^{(1)}, n=0,1, \ldots, k-1$, and (8) has at least one root with positive real part.

That is to say, under those conditions when $\tau_{2}=0, \tau_{1} \in\left[0, \tau_{1}^{0}\right)$, system (4) is asymptotically stable, and system (4) undergoes a Hopf bifurcation when $\tau_{1}=\tau_{1}^{0}$.

Then, we consider stable interval for $\tau_{1}$ in which all roots of (8) have negative real parts, regarding $\tau_{2}>0$ as a parameter.

Lemma 4. If all roots of (8) have negative real parts, then there exists a $\tau_{2}^{0}\left(\tau_{1}\right)>0$, such that when $\tau_{2} \in\left[0, \tau_{2}^{0}\left(\tau_{1}\right)\right)$ all roots of (7) have negative real parts.

Proof. All roots of (8) have negative real parts which means that system (4) is stable when $\tau_{2}=0$. In what follows, we consider (4) with fixed $\tau_{1}$ in its stable interval, regarding $\tau_{2}$ as a parameter. Let iv $(v>0)$ be a root of (4); then we obtain

$$
\begin{aligned}
& -v^{2}+A\left(\cos v \tau_{2}-i \sin v \tau_{2}\right) v i+B k\left(\cos v \tau_{2}-i \sin v \tau_{2}\right) \\
& +C d\left(\cos v \tau_{1}-i \sin v \tau_{1}\right) \\
& +C k\left(\cos v\left(\tau_{1}+\tau_{2}\right)-i \sin v\left(\tau_{1}+\tau_{2}\right)\right)=0 .
\end{aligned}
$$

Suppose that $F(v)=v^{2}-A v i \cos v \tau_{2}-A v \sin v \tau_{2}-B k \cos v \tau_{2}+$ $B k i \sin v \tau_{2}-C d \cos v \tau_{2}+C d i \sin v \tau_{2}-C k \cos v\left(\tau_{1}+\tau_{2}\right)+$ $C k i \sin v\left(\tau_{1}+\tau_{2}\right)$. Since $F(0)=-(B k+C d+C k)<0$ and $F(+\infty)=+\infty$, then (19) has at least one positive root. Without loss of generality, the roots of (19) are defined by $v_{1}, v_{2}, \ldots, v_{k}$. For every $v_{i}(i=1,2, \ldots, k)$, there exists a sequence $\left\{\tau_{2 i}^{(j)} \mid j=1,2, \ldots\right\}$, such that (19) holds. The expression of $\tau_{2 i}^{(j)}$ and $v_{i}$ can be derived by (19) for fixed $\tau_{1}$; we will calculate them directly by the use of Mathematica software in Section 4; here we omit them. Let $\tau_{2}^{0}=\left\{\min \tau_{2 i}^{j} \mid\right.$ $i=1,2, \ldots, k, j=1,2, \ldots\}$ and let $v_{0}$ be the positive and simple root of (19) when $\tau_{2}=\tau_{2}^{0}$. When $\tau_{2}=\tau_{2}^{0}$, (19) has a pair of purely imaginary roots $\pm i v^{*}$ for $\tau_{1} \in\left[0, \tau_{1}^{0}\right)$. Then, as $\tau_{2}$ varies, the sum of the multiplicities of zeros in the open right half-plane can change only if a zero appears on or crosses the imaginary axis. In what follows, we assume that (H6) $\left[d \operatorname{Re} \lambda\left(\tau_{2}\right) / d \tau_{2}\right]_{\tau_{2}=\tau_{2 i}^{j}} \neq 0$. Therefore, by the general Hopf bifurcation theorem for FDEs in Wei and Ruan [10], 
we get that when $\tau_{2} \in\left[0, \tau_{2}^{0}\right)$ all roots of (7) have negative real parts.

Applying the discussion above and noticing that all roots of (8) have negative real parts, we know that there exist $\tau_{2}^{0}>0$ such that all roots of $(7)$ with $\tau_{2} \in\left[0, \tau_{2}^{0}\right.$ ) have negative real parts. The proof is complete.

Summarizing the above lemmas and literature of Hale [11], we obtain the following sufficient conditions for all characteristic roots of (8) to have negative real parts.

Theorem 5. Suppose that (H6) holds and either (H1) or (H2) is satisfied.

(i) If (H3) holds, then for any $\tau_{1} \in\left[0, \tau_{1,0}^{(1)}\right)$ there exists a $\tau_{2}^{0}\left(\tau_{1}\right)>0$ such that when $\tau_{2}^{0} \in\left[0, \tau_{2}\left(\tau_{1}\right)\right)$, all roots of (7) have negative real parts.

(ii) If (H4) holds, then for any $\tau_{1} \in \cup_{n=-1}^{k-1}\left(\tau_{1, n}^{(2)}, \tau_{1, n+1}^{(1)}\right)$ there exists a $\tau_{2}\left(\tau_{1}\right)>0$, such that when $\tau_{1} \in\left[0, \tau_{1,0}^{(1)}\right)$ all roots of (7) have negative real parts, where $\tau_{1, j}^{(1)}$ and $\tau_{1, j}^{(2)}$ are defined by (10).

(iii) If (H5) holds, then for any $\tau_{1} \geq 0$, there exists a $\tau_{2}\left(\tau_{1}\right)>$ 0 , such that when $\tau_{2} \in\left[0, \tau_{2}\left(\tau_{1}\right)\right)$ all roots of $(7)$ have negative real parts.

That is to say, under the conditions that $\tau_{1}$ is stable interval, there exist a $\tau_{2}$ (depend on $\tau_{1}$ ) such that when $\tau_{2} \in\left[0, \tau_{2}^{0}\right.$ ), system (4) is asymptotically stable, and system (4) undergoes a Hopf bifurcation when $\tau_{2}=\tau_{2 i}^{(j)}, i=1,2, \ldots, k ; j=1,2 \ldots$.

\section{Direction and Stability of the Bifurcating Periodic Solutions}

In the previous section, we obtain the conditions under which a family of periodic solutions bifurcate from the steady state and the equilibrium loses its stability when $\tau_{2}=\tau_{2 i}^{(j)}, i=$ $1,2, \ldots, k ; j=1,2, \ldots$ for fixed $\tau_{1}$, and the relationship between $\tau_{1}$ and $\tau_{2}$ can be derived by (19). Throughout this section, by using techniques of the normal form and center manifold theory due to Hale [11], we derive the algorithm for determining the direction of the Hopf bifurcations and the stability of the bifurcating periodic solutions at critical values on the center manifold.

Since the analysis is local, we regard $\tau_{2}=\tau_{2}^{0}+\gamma, \gamma \in$ $R$ as bifurcation parameter. Choosing the space as $C=$ $C\left(\left[-\tau_{2}^{0}, 0\right], R^{2}\right)$ and $u_{t}=u(t+\theta) \in C$ for $\theta \in\left[-\tau_{2}^{0}, 0\right]$, system (3) is transformed into FDE as

$$
\dot{u}(t)=L_{\gamma}(\phi)+F(\phi, \gamma)
$$

with

$$
\begin{gathered}
L_{\gamma}(\phi)=B_{1} \phi(0)+B_{2} \phi\left(-\tau_{1}\right)+B_{3} \phi\left(-\tau_{2}\right), \\
F(\phi, \gamma)=\left(\begin{array}{c}
0 \\
\mu b \phi_{1}^{2}(0) \phi_{2}\left(-\tau_{2}\right)+2 \mu\left(P_{0}+\beta\right) \phi_{1}(0) \phi_{2}(0)
\end{array}\right),
\end{gathered}
$$

where $B_{1}=\left(\begin{array}{cc}0 & d \\ -\mu b_{0} & 0\end{array}\right), B_{2}=\left(\begin{array}{cc}0 & 0 \\ -\mu a & 0\end{array}\right)$, and $B_{3}=\left(\begin{array}{cc}0 & k \\ 0 & 2 \mu\left(P_{0}+\beta\right)^{2}+C_{0}\end{array}\right)$. Obviously, $L_{\gamma}(\phi)$ is continuous linear function mapping $C\left(\left[-\tau_{2}^{0}, 0\right], R^{2}\right)$ into $R^{2}$. By the Riesz representation theorem, there exists a matrix whose elements are bounded variation functions $\eta(\theta, \gamma)$ in $\theta \in\left[-\tau_{2}^{0}, 0\right]$ such that

$$
L_{\gamma} \phi=\int_{-\tau_{2}^{0}}^{0} d \eta(\theta, \gamma) \phi(\theta), \quad \text { for } \phi \in C .
$$

In fact, we choose

$$
\eta(\theta, \gamma)=B_{1} \delta(\theta)+B_{2} \delta\left(\theta+\tau_{1}\right)+B_{3} \delta\left(\theta+\tau_{2}\right),
$$

where $\delta(\theta)$ is a delta function.

For $\phi \in C^{\prime}\left(\left[-\tau_{2}^{0}, 0\right], R^{2}\right)$ the operators $A$ and $R$ are defined as

$$
\begin{aligned}
& A(\mu) \phi(\theta)= \begin{cases}\frac{d \phi(\theta)}{d \theta}, & \theta \in\left[-\tau_{2}^{0}, 0\right), \\
\int_{-\tau_{2}^{0}}^{0} d(\eta(t, \mu) \phi(t)), & \theta=0,\end{cases} \\
& R(\mu) \phi(\theta)= \begin{cases}0, & \theta \in\left[-\tau_{2}^{0}, 0\right), \\
f(\gamma, \theta), & \theta=0 .\end{cases}
\end{aligned}
$$

Let $\psi \in C^{\prime}\left[0, \tau_{2}^{0}\right]$; the adjoint operator $A^{*}(0)$ corresponding to $A(0)$ is defined as follows:

$$
A^{*} \psi(s)= \begin{cases}-\frac{d \psi(s)}{d s}, & s \in\left(0, \tau_{2}^{0}\right], \\ \int_{-\tau_{2}^{0}}^{0} d\left(\eta^{T}(t, 0) \psi(-t)\right), & s=0 .\end{cases}
$$

Then system (20) can be written in the following form: $\dot{u}_{t}=$ $A(\alpha) u_{t}+R(\alpha) u_{t}$, where $u_{t}=u(t+\theta)$ for $\theta \in[-1,0)$.

For $\phi \in C^{\prime}\left(\left[-\tau_{2}^{0}, 0\right], R^{2}\right)$ and $\psi \in C^{\prime}\left[0, \tau_{2}^{0}\right]$, define the adjoint bilinear:

$$
\langle\psi, \phi\rangle=\bar{\psi}(0) \phi(0)-\int_{-\tau_{2}^{0}}^{0} \int_{\varepsilon=0}^{\theta} \bar{\psi}(\xi-\theta) d \eta(\theta) \phi(\xi) d \xi,
$$

where $\eta(\theta)=\eta(\theta, 0)$.

Proposition 6. Let $q(\theta)$ and $q^{*}(s)$ be eigenvectors of $A$ and $A^{*}$ corresponding to $i \omega_{0}$ and $-i \omega_{0}$, respectively, satisfying $\left\langle q^{*}, q\right\rangle=$ 1 and $\left\langle q^{*}, \bar{q}\right\rangle=0$. Then

$$
\begin{gathered}
q(\theta)=\left(q_{1}, q_{2}\right)^{T} e^{i \omega_{0} \theta}=\left(d+k e^{-i \omega_{0} \tau_{2}}, i \omega\right)^{T} e^{i \omega_{0} \theta}, \\
q^{*}(s)=\bar{D}\left(q_{1}^{*}, q_{2}^{*}\right) e^{-i \omega_{0} s}=\left(\mu b_{0}+\mu a e^{i \omega_{0} \tau_{1}}, i \omega\right) e^{-i \omega_{0} s},
\end{gathered}
$$

where

$$
\begin{aligned}
\bar{D}= & {\left[\left(q_{1} \bar{q}_{1}^{*}+q_{2} \bar{q}_{2}^{*}\right)+\tau_{1}^{0}\left[-\mu a e^{-i \omega_{0} \tau_{1}^{0}} q_{1} \bar{q}_{2}^{*}\right]\right.} \\
& \left.+\left[k q_{2} \bar{q}_{1}^{*}+\left[2 \mu b\left(P_{0}+\beta\right)^{2}+C_{0}\right] \bar{q}_{2}^{*} q_{2}\right] \tau_{2}^{0} e^{-i \omega_{0} \tau_{2}^{0}}\right]^{-1} .
\end{aligned}
$$


Proof. We assume that $q(\theta)=\left(q_{1}, q_{2}\right)^{T} e^{i \omega_{0} \theta}$ is the eigenvector of $A(0)$ corresponding to $i \omega$ and $q^{*}(s)=\bar{D}\left(q_{1}^{*}, q_{2}^{*}\right)^{T} e^{-i \omega_{0} s}$ is the eigenvector of $A^{*}(0)$ corresponding to $-i \omega$. It follows from the definition of $A(0), A^{*}(0),(22)$, and (23) that we have $A q(0)=\int_{-\tau_{2}^{0}}^{0} d(\eta(t, \mu) \phi(t))=i \omega_{0} q(0)$ and $A^{*} q(0)=$ $\int_{-\tau_{2}^{0}}^{0} d\left(\eta^{T}(t, \mu) \phi(-t)\right)=-i \omega_{0} q(0)$; we have

$$
\begin{aligned}
& {\left[i \omega_{0} I-\left(B_{1}+B_{2} e^{-i \omega_{0} \tau_{1}}+B_{3} e^{-i \omega_{0} \tau_{2}}\right)\right] q(0)=0,} \\
& {\left[-i \omega_{0} I-\left(B_{1}+B_{2} e^{i \omega_{0} \tau_{1}}+B_{3} e^{i \omega_{0} \tau_{2}}\right)\right] q^{*}(0)=0,}
\end{aligned}
$$

where $I$ is identity matrix; that is,

$$
\begin{aligned}
& \left(\begin{array}{cc}
i \omega_{0} & -\left(d+k e^{-i \omega_{0} \tau_{2}}\right) \\
\mu\left(b_{0}+a e^{-i \omega_{0} \tau_{1}}\right) & i \omega_{0}-\mu\left[b\left(P_{0}+\beta\right)+C_{0}\right] e^{-i \omega_{0} \tau_{2}}
\end{array}\right) \\
& \times\left(\begin{array}{c}
q_{1} \\
q_{2}
\end{array}\right)=0, \\
& \left(\begin{array}{cc}
-i \omega_{0} & \mu\left(b_{0}+a e^{i \omega_{0} \tau_{1}}\right) \\
-\left(d+k e^{i \omega_{0} \tau_{2}}\right) & -i \omega_{0}-\mu\left[b\left(P_{0}+\beta\right)+C_{0}\right] e^{i \omega_{0} \tau_{2}}
\end{array}\right) \\
& \times\left(\begin{array}{c}
q_{1}^{*} \\
q_{2}^{*}
\end{array}\right)=0 .
\end{aligned}
$$

By direct computation and considering $q(\theta)=q(0) e^{i \omega \theta}$, $q^{*}(s)=q^{*}(0) e^{-i \omega s}$, we obtain $q(\theta)$ and $q^{*}(s)$. Now, we calculate $\left\langle q^{*}, q\right\rangle$ as follows:

$$
\begin{aligned}
& \left\langle q(s)^{*}, q(\theta)\right\rangle \\
& =\bar{D}\left\{\left(\bar{q}_{1}^{*}, \bar{q}_{2}^{*}\right)\left(\begin{array}{l}
q_{1} \\
q_{2}
\end{array}\right)\right. \\
& \left.\quad-\int_{-\tau_{2}^{0}}^{0} \int_{\varepsilon=0}^{\theta}\left(\begin{array}{l}
\bar{q}_{1}^{*} \\
\bar{q}_{2}^{*}
\end{array}\right) e^{T}-i \omega_{0}(\varepsilon-\theta) d \eta(\theta)\left(\begin{array}{l}
q_{1} \\
q_{2}
\end{array}\right) e^{i \omega_{0} \varepsilon} d \varepsilon\right\} \\
& =\bar{D}\left\{\left(q_{1} \bar{q}_{1}^{*}+q_{2} \bar{q}_{2}^{*}\right)-\int_{-\tau_{2}^{0}}^{0}\left(\begin{array}{l}
\bar{q}_{1}^{*} \\
\bar{q}_{2}^{*}
\end{array}\right)^{T} \theta e^{i \omega_{0} \theta} d \eta(\theta)\left(\begin{array}{l}
q_{1} \\
q_{2}
\end{array}\right)\right\} \\
& =\bar{D}\left\{\left(q_{1} \bar{q}_{1}^{*}+q_{2} \bar{q}_{2}^{*}\right)+\tau_{1}^{0}\left[-\mu a e^{-i \omega_{0} \tau_{1}^{0}} q_{1} \bar{q}_{2}^{*}\right]\right. \\
& \left.+\left[k q_{2} \bar{q}_{1}^{*}+\left[2 \mu b\left(P_{0}+\beta\right)^{2}+C_{0}\right] \bar{q}_{2}^{*} q_{2}\right] \tau_{2}^{0} e^{-i \omega_{0} \tau_{2}^{0}}\right\}
\end{aligned}
$$$$
=1 \text {. }
$$

Since $\langle\psi, A \phi\rangle=\left\langle A^{*} \psi, \phi\right\rangle$, we get

$$
i \omega\left\langle q^{*}, \bar{q}\right\rangle=\left\langle A^{*} q^{*}, \bar{q}\right\rangle=\left\langle-i \omega_{0} q^{*}, \bar{q}\right\rangle=i \omega_{0}\left\langle q^{*}, \bar{q}\right\rangle .
$$

Therefore, $\left\langle q^{*}, \bar{q}\right\rangle=0$. This completes the proof.

Then, we construct the coordinates of the center manifold $C_{0}$ at $\gamma=0$. Let

$$
z(t)=\left\langle q^{*}, u_{t}\right\rangle, \quad W(t, \theta)=u_{t}(\theta)-2 \operatorname{Re}\{z(t) q(\theta)\} .
$$

On the center manifold $C_{0}$, we have

$$
W(t, \theta)=W(z(t), \bar{z}(t), \theta),
$$

where

$$
\begin{aligned}
W(z, \bar{z}, \theta)= & W_{20}(\theta) \frac{z^{2}}{2}+W_{11}(\theta) z \bar{z} \\
& +W_{02} \frac{\bar{z}^{2}}{2}+W_{30} \frac{z^{3}}{6}+\cdots,
\end{aligned}
$$

and $z$ and $\bar{z}$ are local coordinates for the center manifold $C_{0}$ in the direction of $q$ and $q^{*}$, respectively. Since $\gamma=0$, we have

$$
\begin{aligned}
z^{\prime}(t) & =i \omega_{0} z(t)+\left\langle q^{*}(\theta), f(W+2 \operatorname{Re}\{z(t) q(\theta)\})\right\rangle \\
& =i \omega_{0} z(t)+\bar{q}^{*}(0) f(W(z, \bar{z}, 0)+2 \operatorname{Re}\{z(t) q(0)\}) \\
& \triangleq i \omega_{0} z(t)+\bar{q}^{*}(0) f_{0}(z, \bar{z}),
\end{aligned}
$$

where

$$
f_{0}(z, \bar{z})=f_{z^{2}} \frac{z^{2}}{2}+f_{\bar{z}^{2}} \frac{\bar{z}^{2}}{2}+f_{z \bar{z}} z \bar{z}+\cdots
$$

We rewrite in abbreviated form as

$$
z^{\prime}(t)=i \omega_{0} z+g(z, \bar{z}),
$$

where

$$
\begin{aligned}
g(z, \bar{z}) & =\bar{q}^{*}(0) f_{0}(z, \bar{z}) \\
& =g_{20} \frac{z^{2}}{2}+g_{11} z \bar{z}+g_{02} \frac{\bar{z}^{2}}{2}+g_{21} \frac{z^{2} \bar{z}}{2}+\cdots .
\end{aligned}
$$

By (20) and (38), we obtain

$$
\begin{aligned}
\dot{W} & =\dot{u}_{t}-\dot{z} q-\dot{\bar{z}} \bar{q} \\
& = \begin{cases}A W-2 \operatorname{Re} \bar{q}^{*}(0) f_{0} q(\theta), & \theta \in\left[-\tau_{2}^{0}, 0\right], \\
A W-2 \operatorname{Re} \bar{q}^{*}(0) f_{0} q(\theta)+f_{0}, & \theta=0\end{cases} \\
& \triangleq A W+H(z, \bar{z}, \theta),
\end{aligned}
$$

where

$$
H(z, \bar{z}, \theta)=H_{20}(\theta) \frac{z^{2}}{2}+H_{11}(\theta) z \bar{z}+H_{02}(\theta) \frac{\bar{z}^{2}}{2}+\cdots .
$$

Substituting (26) and (38) into $\dot{W}=W_{z} \dot{z}+W_{\bar{z}} \dot{\bar{z}}$ on the center manifold $C_{0}$ and comparing the coefficients we get

$$
\begin{gathered}
\left(A-2 i \omega_{0} I\right) W_{20}(\theta)=-H_{20}(\theta), \quad A W_{11}(\theta)=-H_{11}(\theta), \\
\left(A+2 i \omega_{0} I\right) W_{02}(\theta)=-H_{02}(\theta) .
\end{gathered}
$$

Comparing the coefficients with (41) gives that

$$
\begin{aligned}
& H_{20}(\theta)=-g_{20} q(\theta)-\bar{g}_{02} \bar{q}(\theta), \\
& H_{11}(\theta)=-g_{11} q(\theta)-\bar{g}_{11} \bar{q}(\theta) .
\end{aligned}
$$


From (42), (43), and the definition of $A$, we can derive the following equation:

$$
\begin{gathered}
\dot{W}_{20}(\theta)=2 i \omega_{0} W_{20}(\theta)+g_{20} q(\theta)+\bar{g}_{20} \bar{q}(\theta), \\
\dot{W}_{11}(\theta)=g_{11} q(\theta)+\bar{g}_{11} \bar{q}(\theta) .
\end{gathered}
$$

Solving for $W_{20}(\theta)$ and $W_{11}(\theta)$, we get

$$
\begin{gathered}
W_{20}(\theta)=\frac{i g_{20}}{\omega_{0}} q(0) e^{i \omega_{0} \theta}-\frac{\bar{g}_{02}}{3 i \tau_{0} \omega_{0}} \bar{q}(0) e^{-i \omega_{0} \theta}+E_{1} e^{2 i \omega_{0} \theta}, \\
W_{11}(\theta)=-\frac{i g_{11}}{\omega_{0}} q(0) e^{i \omega_{0} \theta}+\frac{i \bar{g}_{11}}{\omega_{0}} \bar{q}(0) e^{-i \omega_{0} \theta}+E_{2},
\end{gathered}
$$

where $E_{1}=\left(E_{1}^{(1)}, E_{1}^{2}\right) \in R^{2}, E_{2}=\left(E_{2}^{(1)}, E_{2}^{2}\right) \in R^{2}$ are two constant vectors, which can be determined by setting $\theta=0$ in $H$.

$\operatorname{By}(33), \phi_{t}(\theta)=\left[u_{1}\left(t-\tau_{1}\right), u_{2}\left(t-\tau_{2}\right)\right]=W(t, \theta)+z q(\theta)+$ $\bar{z} \bar{q}(\theta)$, and noticing $q(\theta)=\left(q_{1}, q_{2}\right)^{T} e^{i \omega_{0} \theta}$ we have

$$
\begin{aligned}
\phi_{t}(\theta)= & z\left(\begin{array}{c}
d+k e^{-i \omega_{0} \tau_{2}^{0}} \\
i \omega
\end{array}\right) e^{-i \omega_{0} \theta} \\
& +\bar{z}\left(\begin{array}{c}
d+k e^{i \omega_{0} \tau_{2}^{0}} \\
-i \omega
\end{array}\right) e^{-i \omega_{0} \theta}+W(t, \theta) .
\end{aligned}
$$

Then it is easy to obtain

$$
\begin{aligned}
\phi_{1}(0) & =z\left(d+k e^{-i \omega_{0} \tau_{2}^{0}}\right)+\bar{z}\left(d+k e^{i \omega_{0} \tau_{2}^{o}}\right)+W^{(1)}(0), \\
\phi_{2}(0) & =z \omega i+\bar{z}(-i \omega)+W^{(2)}(0), \\
\phi_{2}\left(t-\tau_{2}^{0}\right) & =z \omega i e^{-i \omega_{0} \tau_{2}^{0}}-\bar{z} e^{i \omega_{0} \tau_{2}^{0}}+W^{2}\left(t-\tau_{2}^{0}\right),
\end{aligned}
$$

where

$$
\begin{aligned}
& W^{(1)}(0)=W_{20}^{(1)}(0) \frac{z^{2}}{2}+W_{11}^{(1)}(0) z \bar{z} \\
& +W_{02}^{(1)}(0) \frac{\bar{z}}{2}+o\left(|z, \bar{z}|^{3}\right) \\
& W^{(2)}(0)=W_{20}^{(2)}(0) \frac{z^{2}}{2}+W_{11}^{(2)}(0) z \bar{z} \\
& +W_{02}^{(2)}(0) \frac{\bar{z}}{2}+o\left(|z, \bar{z}|^{3}\right), \\
& W^{(2)}\left(t-\tau_{2}\right)=W_{20}^{(2)}\left(-\tau_{2}^{0}\right) \frac{z^{2}}{2}+W_{11}^{(2)}\left(-\tau_{2}^{0}\right) z \bar{z} \\
& +W_{02}^{(2)}\left(-\tau_{2}^{0}\right) \frac{\bar{z}}{2}+o\left(|z, \bar{z}|^{3}\right), \\
& f_{0}(z, \bar{z})=\left(\begin{array}{c}
0 \\
\mu b \phi_{1}^{2}(0) \phi_{2}\left(-\tau_{2}^{0}\right)+2 \mu\left(P_{0}+\beta\right) \phi_{1}(0) \phi_{2}(0)
\end{array}\right) .
\end{aligned}
$$

Thus, from (39), it follows that

$$
\begin{aligned}
& g(z, \bar{z})=\bar{q}^{*}(0) f_{0}(z, \bar{z})=\bar{D}\left(\bar{q}_{1}^{*} \bar{q}_{2}^{*}\right) \\
& \times\left(\begin{array}{c}
0 \\
\mu b \phi_{1}^{2}(0) \phi_{2}\left(-\tau_{2}^{0}\right)+2 \mu\left(P_{0}+\beta\right) \phi_{1}(0) \phi_{2}(0)
\end{array}\right) \\
& =\bar{D} \bar{q}_{2}^{*}\left[\mu \phi_{1}^{2}(0) \phi_{2}\left(-\tau_{2}^{0}\right)+2 \mu\left(P_{0}+\beta\right) \phi_{1}(0) \phi_{2}(0)\right] \\
& =\bar{D}\left\{2 \mu\left(P_{0}+\beta\right) \bar{q}_{2}^{*} q_{1} q_{2} z^{2}+2 \mu\left(P_{0}+\beta\right)\right. \\
& \times \bar{q}_{2}^{*}\left(q_{1} \bar{q}_{2}+\bar{q}_{1} q_{2}\right) z \bar{z}+2 \mu\left(P_{0}+\beta\right) \bar{q}_{2}^{*} \bar{q}_{1} \bar{q}_{2} \bar{z}^{2} \\
& +\left[\mu b \bar{q}_{2}^{*}\left(q_{1}^{2} \bar{q}_{2} e^{i \omega_{0} \tau_{2}^{0}}+2 q_{1} \bar{q}_{1} q_{2} e^{-i \omega_{0} \tau_{2}^{0}}\right)\right. \\
& +2 \mu\left(P_{0}+\beta\right) \bar{q}_{2}^{*} \\
& \times\left(q_{2} W_{11}^{(1)}(0)+q_{1} W_{11}^{(2)}(0)\right. \\
& \left.\left.\left.+\frac{\bar{q}_{2} W_{20}^{(1)}(0)+\bar{q}_{1} W_{20}^{(2)}(0)}{2}\right)\right] z^{2} \bar{z}\right\} \\
& +o\left(\phi^{4}\right) \text {. }
\end{aligned}
$$

Comparing the coefficient with (39), we have

$$
\begin{gathered}
g_{20}=4 \bar{D} \mu\left(P_{0}+\beta\right) \bar{q}_{2}^{*} q_{1} q_{2}, \\
g_{11}=4 \bar{D} \mu\left(P_{0}+\beta\right) \bar{q}_{2}^{*} \operatorname{Re}\left(q_{1} \bar{q}_{2}\right), \\
g_{02}=4 \bar{D} \mu\left(P_{0}+\beta\right) \bar{q}_{2}^{*} \bar{q}_{1} \bar{q}_{2}, \\
g_{21}=2 \bar{D}\left[\mu b \bar{q}_{2}^{*}\left(q_{1}^{2} \bar{q}_{2} e^{i \omega_{0} \tau_{2}^{0}}+2 q_{1} \bar{q}_{1} q_{2} e^{-i \omega_{0} \tau_{2}^{0}}\right)\right. \\
+2 \mu\left(P_{0}+\beta\right) \bar{q}_{2}^{*} \\
\times\left(q_{2} W_{11}^{(1)}(0)+q_{1} W_{11}^{(2)}(0)\right. \\
\left.\left.+\frac{\bar{q}_{2} W_{20}^{(1)}(0)+\bar{q}_{1} W_{20}^{(2)}(0)}{2}\right)\right] .
\end{gathered}
$$

From (40), we get that $H(z, \bar{z}, 0)=-2 \operatorname{Re} \bar{q}^{*}(0) f_{0} q(0)+f_{0}=$ $-2 \operatorname{Re}(g q(0))+f_{0}=-g q(0)-\bar{g} \bar{q}(0)+f_{0}$; that is,

$$
H_{20}(0)=-g_{20} q(0)-\bar{g}_{20} \bar{q}(0)+\left(\begin{array}{c}
0 \\
4 \mu\left(P_{0}+\beta\right) q_{1} q_{2}
\end{array}\right),
$$

$H_{11}(0)=-g_{11} q(0)-\bar{g}_{11} \bar{q}(0)+\left(\begin{array}{c}0 \\ 2\left(P_{0}+\beta\right)\left(q_{1} \bar{q}_{2}+\bar{q}_{1} q_{2}\right)\end{array}\right)$. 
By the definition of $A$ and (42) we have

$$
\begin{gathered}
\int_{-\tau_{2}^{0}}^{0} d \eta(\theta) W_{20}(\theta)=2 i \omega_{0} W_{20}-H_{20}(0) \\
\int_{-\tau_{2}^{0}}^{0} d \eta(\theta) W_{11}(\theta)=-H_{11}(0) .
\end{gathered}
$$

Notice that

$$
\begin{gathered}
\left(i \omega_{0} I-\int_{-\tau_{2}^{0}}^{0} d \eta(\theta) e^{i \omega_{0} \theta}\right) q(0)=0, \\
\left(-i \omega_{0} I-\int_{-\tau_{2}^{0}}^{0} d \eta(\theta) e^{i \omega_{0} \theta}\right) \bar{q}(0)=0 .
\end{gathered}
$$

$$
\begin{gathered}
E_{1}^{(1)}=\frac{\left[4 \mu\left(P_{0}+\beta\right) q_{1} q_{2}\right]\left(d+k e^{-2 i \omega_{0} \tau_{2}^{0}}\right)}{\left[\mu b_{0}+\mu a e^{-2 i \omega_{0} \tau_{1}^{0}}\right]\left(d+k e^{-2 i \omega_{0} \tau_{2}^{0}}\right)-2 i \omega_{0}\left[\mu\left[b\left(P_{0}+\beta\right)^{2}+C_{0}\right] e^{-2 i \omega_{0} \tau_{2}^{0}}-2 i \omega_{0}\right]}, \\
E_{1}^{(2)}=\frac{-6 i \omega_{0} \mu\left(P_{0}+\beta\right) q_{1} q_{2}}{-\left[\mu b_{0}+\mu a e^{-2 i \omega_{0} \tau_{1}^{0}}\right]\left(d+k e^{-2 i \omega_{0} \tau_{2}^{0}}\right)+2 i \omega_{0}\left[\mu\left[b\left(P_{0}+\beta\right)^{2}+C_{0}\right] e^{-2 i \omega_{0} \tau_{2}^{0}}-2 i \omega_{0}\right]}, \\
E_{2}^{(1)}=\frac{2\left(P_{0}+\beta\right)\left(q_{1} \bar{q}_{2}+\bar{q}_{1} q_{2}\right)}{\mu\left(b_{0}\right)-a}, \quad E_{2}^{(2)}=0 .
\end{gathered}
$$

Substituting (45) and (52) into (51), we obtain

$$
\begin{aligned}
& \left(\begin{array}{cc}
2 i \omega_{0} & -d-k e^{2 i \omega_{0} \tau_{2}^{0}} \\
\mu b_{0}+\mu a e^{-2 i \omega_{0} \tau_{1}^{0}} & 2 i \omega_{0}-\mu\left[b\left(P_{0}+\beta\right)^{2}+C_{0}\right] e^{2 i \omega_{0} \tau_{2}^{0}}
\end{array}\right) E_{1} \\
& =\left(\begin{array}{c}
0 \\
4 \mu\left(P_{0}+\beta\right) q_{1} q_{2}
\end{array}\right) \\
& \left.\left(\begin{array}{cc}
0 & -d-k \\
\mu\left(b_{0}+a\right)-\mu\left[b\left(P_{0}+\beta\right)^{2}+C_{0}\right.
\end{array}\right]\right) E_{2} \\
& =\left(\begin{array}{c}
0 \\
2\left(P_{0}+\beta\right)\left(q_{1} \bar{q}_{2}+\bar{q}_{1} q_{2}\right)
\end{array}\right) .
\end{aligned}
$$

By direct computation, we obtain
Thus, we can compute the following values which determine the properties of bifurcating periodic solutions at the critical value $\tau_{2}^{0}$ :

$$
\begin{gathered}
C_{1}(0)=\frac{i}{2 \tau_{2}^{0} \omega_{0}}\left(g_{20} g_{11}-2\left|g_{11}\right|-\frac{1}{3}\left|g_{02}\right|^{2}\right)+\frac{g_{21}}{2}, \\
\mu_{2}=-\frac{\operatorname{Re}\left\{C_{1}(0)\right\}}{\operatorname{Re}\left\{\lambda^{\prime}\left(\tau_{2}^{0}\right)\right\}}, \\
\beta_{2}=2 \operatorname{Re}\left\{C_{1}(0)\right\}, \\
T_{2}=-\frac{\operatorname{Im}\left\{C_{1}(0)\right\}+\mu_{2}\left(\operatorname{Im}\left\{\lambda^{\prime}\left(\tau_{2}^{0}\right)\right\}\right)}{\omega_{0}} .
\end{gathered}
$$

More specifically (see Hassard et al. [12]), $\mu_{2}$ determines the direction of the Hopf bifurcation: if $\mu_{2}>0\left(\mu_{2}<0\right)$, then the Hopf bifurcation is forward (backward) and the bifurcating periodic solutions exist for $\tau>\tau_{2}^{0}\left(\tau<\tau_{2}^{0}\right) . \beta_{2}$ determines the stability of the bifurcating periodic solutions: the bifurcating periodic solutions are stable (unstable) if $\beta_{2}<0\left(\beta_{2}>0\right) . T_{2}$ determines the period of the bifurcating periodic solutions: the period increases (decreases) if $T_{2}>0\left(T_{2}<0\right)$.

\section{Numerical Simulation}

In order to validate the theoretical analysis, we will present some numerical simulations. We take the following coefficients as an example: $\mu=0.13, b_{0}=10, a=12, d_{0}=60$, $g_{0}=20, \beta=-2, b=1, k=1$, and $d=1$, it is easy to obtain $E^{*}=(1.82,0), \omega_{0} \approx 0.89$, and $\tau_{1}^{0} \approx 0.72$. We choose $\tau_{1}^{0}=0.7$; then from (19) and by means of Mathematica software, we get $v_{0} \approx 2$ and $\tau_{2}^{0} \approx 0.86$. Taking $(x(0), y(0)=(2,2))$ as the initial conditions. By Theorem 5 and the above results, we know equilibrium $E^{*}$ is locally asymptotically stable when $\tau_{2}=0.8<\tau_{2}^{0}$ as is illustrated in Figure 1. When $\tau_{2}>\tau_{2}^{0}$, $E^{*}$ is unstable and periodic solutions occur from $E^{*}$; we take $\tau_{2}=0.9$, the corresponding phase plots are shown in Figure 2 .

Finally, the numerical simulation shows that it is a complex transformation process for the system changes from stable equilibrium to chaos.

\section{Conclusion}

Different from the previous work in $[1,2,4]$, the main contribution of this paper lies in the following aspects. Firstly, we modify the traditional price differential equation model by considering demand function that is settled as a downward opening quadratic function and considering supply and demand functions that are both depending on the price of the past and the present. Then the price differential equation model with two delays is established. Secondly, to study the stability and Hopf bifurcation of system (4), we consider (4) with one delay $\tau_{1}$ and find the stable interval for $\tau_{1}$. In the following, regarding $\tau_{2}$ as a parameter, we obtain the stable interval for $\tau_{2}$. Then we get the stable interval for (4). In addition, we derive the algorithm for determining 


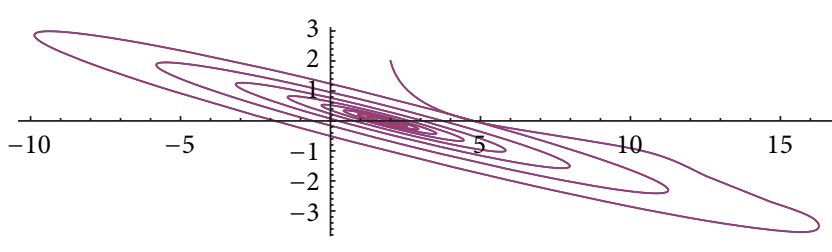

FIgURE 1: Equilibrium $E^{*}$ is locally asymptotically stable when $\tau_{1}=$ $0.7, \tau_{2}=0.8<\tau_{2}^{0}$.

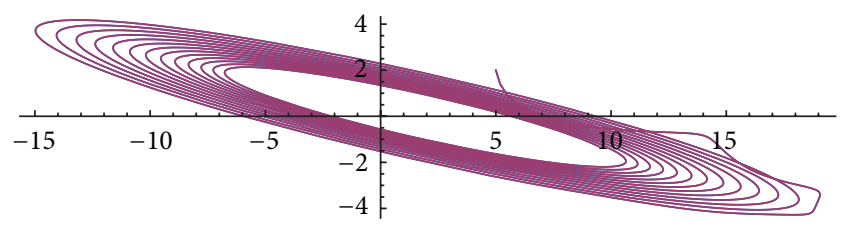

FIGURE 2: Equilibrium $E^{*}$ is unstable when $\tau_{1}=0.7, \tau_{2}=0.9>\tau_{2}^{0}$.

the direction of the Hopf bifurcation and the stability of the bifurcating periodic solutions by using techniques of normal form theory and center manifold method. Lastly, a numerical analysis confirms the effectiveness of our research results. The paper provided the preparative work for further discussion. For instance, we consider that supply cannot increase with the price without limit; the production capacity of enterprises and social resources are limited; then we can modify the supply function as a fractional linear function of price. The results in the paper enrich the toolbox for the qualitative analysis of mathematical economics and business administration.

\section{Conflict of Interests}

The authors declare that there is no conflict of interests regarding the publication of this paper.

\section{Acknowledgment}

The authors are grateful to the referees for their helpful comments and constructive suggestions.

\section{References}

[1] W. Shuhe, "Differential equation model and chaos," Journal of China Science and Technology University, pp. 312-324, 1999.

[2] Z. Xi-fan, C. Xia, and C. Yun-qing, "A qualitative analysis of price model in differential equations of price," Journal of Shenyang Institute of Aeronautical Engineering, vol. 21, no. 1, pp. 83-86, 2004.

[3] S. Banerjee and W. A. Barnett, "Bifurcation analysis of Zellner's marshallain macroeconomic model," Journal of Economic Dynamics and Control, vol. 35, no. 9, pp. 1577-1585.

[4] L. Tanghong and L. Zhenwen, "Hopf bifurcation of price Reyleigh equation with time delay," Journal of Jilin University, vol. 47, no. 3, 2009.

[5] W. Yong and Z. Yanhui, "Stability and Hopf bifurcation of differential equation model of price with time delay," Highlights of Sciencepaper Online, vol. 4, no. 1, 2011.
[6] Y. Zhai, H. Bai, Y. Xiong, and X. Ma, "Hopf bifurcation analysis for the modified Rayleigh price model with time delay," Abstract and Applied Analysis, vol. 2013, Article ID 290497, 6 pages, 2013.

[7] O. I. Adeyemi and L. C. Hunt, "Modelling OECD industrial energy demand: asymmetric price responses and energy saving technical change," Energy Economics, vol. 29, no. 4, pp. 693-709, 2007.

[8] L. Tanghong and Z. Linhua, "Hopf and codimension two bifurcation for the price Rayleigh equation with two time delays," Journal of Jilin University, vol. 50, no. 3, pp. 409-416, 2012.

[9] K. L. Cooke and Z. Grossman, "Discrete delay, distributed delay and stability switches," Journal of Mathematical Analysis and Applications, vol. 86, no. 2, pp. 592-627, 1982.

[10] J. Wei and S. Ruan, "Stability and bifurcation in a neural network model with two delays," Physica D, vol. 130, no. 3-4, pp. 255-272, 1999.

[11] J. Hale, Theory of Functional Differential Equations, Springer, New York, NY, USA, 2nd edition, 1977.

[12] D. D. Hassard, N. D. Kazarinoff, and Y. H. Wan, Theory and Applications of Hopf Bifurcation, Cambridge University Press, Cambridge, UK, 1981. 


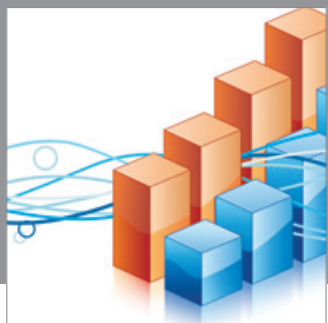

Advances in

Operations Research

mansans

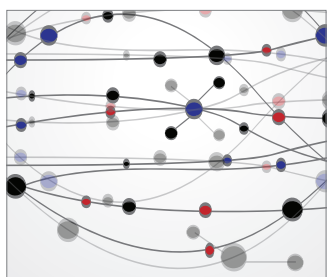

The Scientific World Journal
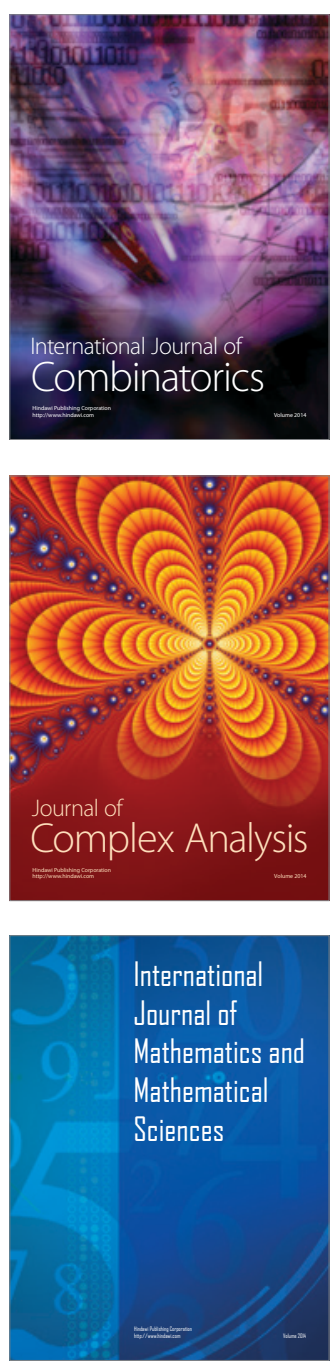
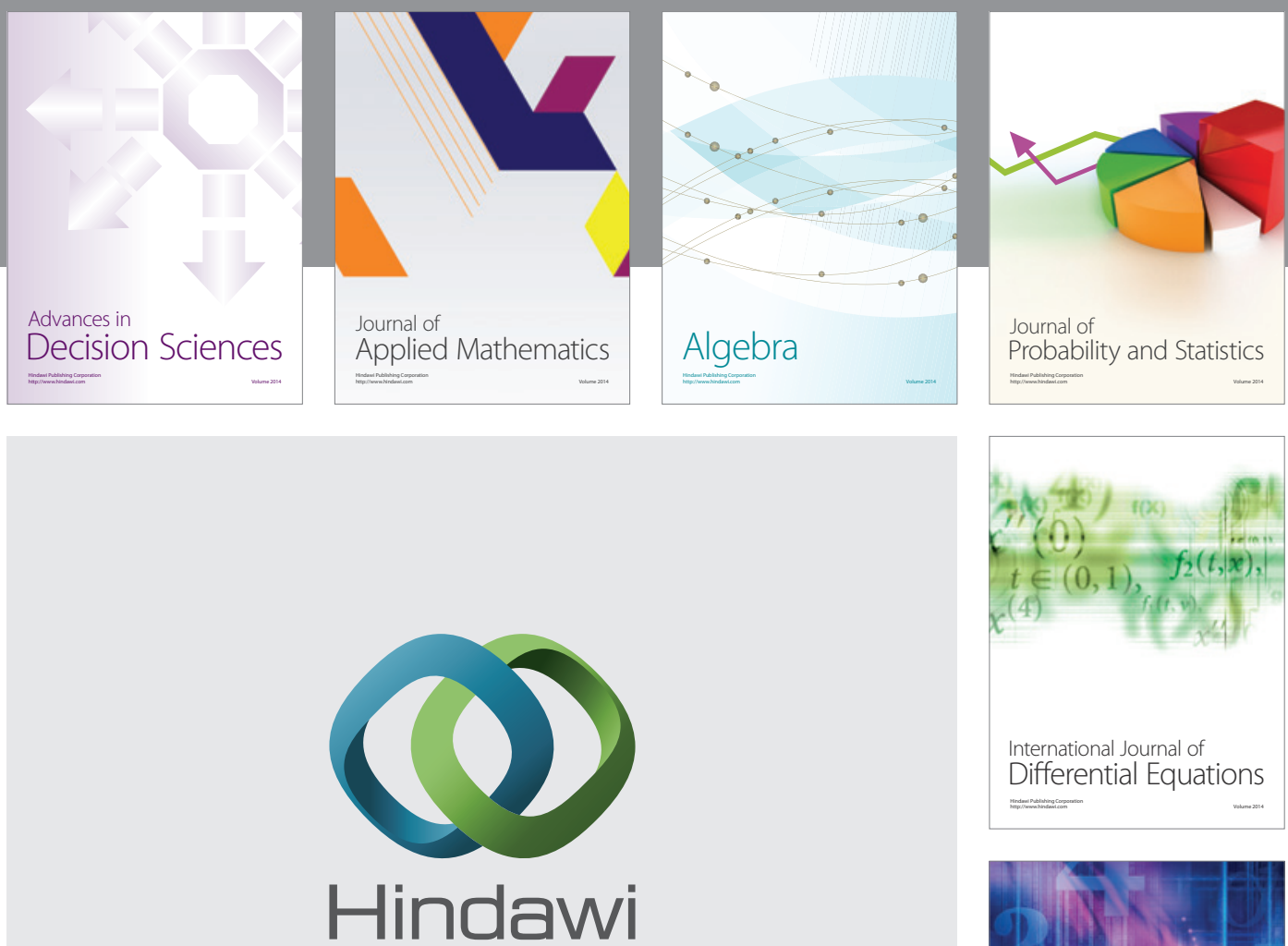

Submit your manuscripts at http://www.hindawi.com
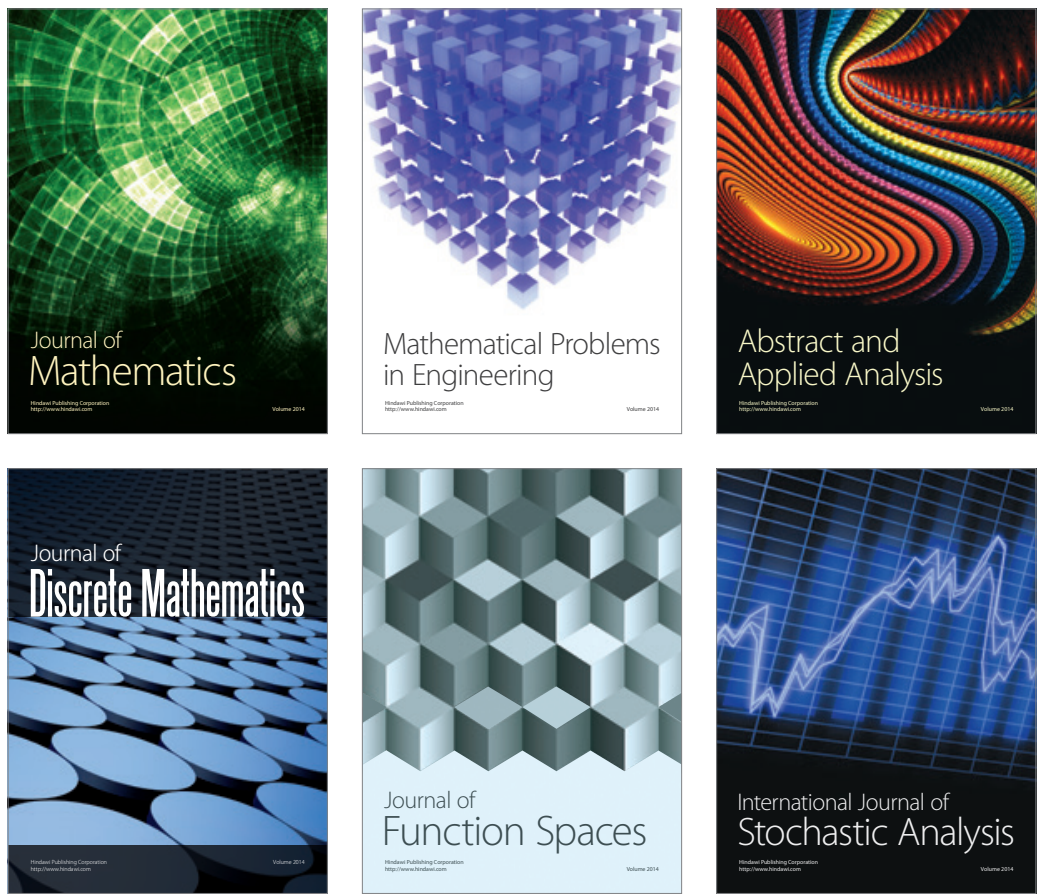

Journal of

Function Spaces

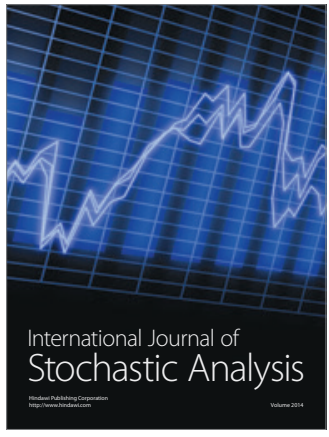

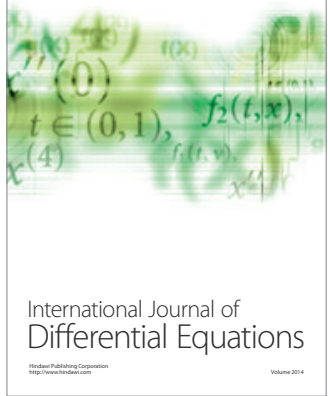
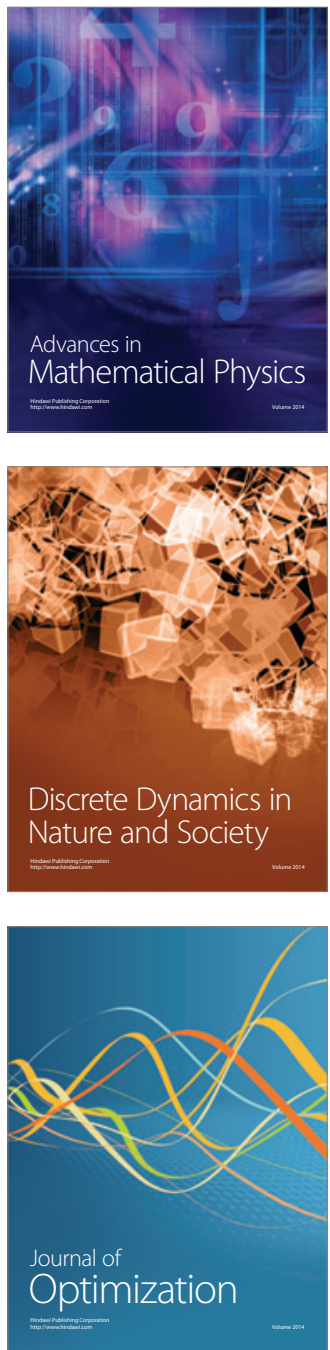